\section{Family in which Duchenne's muscular dystrophy and protan colour blindness are segregating}

SUMMARY A family is recorded in which Duchenne's muscular dystrophy and protan colour blindness are segregating. Of 4 members of the second generation at least one is a recombinant. The lod scores have been calculated and added to those already published.

It seems appropriate to record any families with Duchenne's muscular dystrophy in which colour blindness is segregating. Such families could contribute to future linkage studies. Such a family is shown in the figure.

The dystrophy in the Pe family shown is of typical Duchenne pattern. Onset was at the age of starting to walk, and progress so fast that the youngest is now chairbound at 11 years old. No affected member of the family has survived into his twenties. Colour blindness was detected using Ishihara plates. The $\mathrm{Xg}$ groups gave no linkage information as all members were $\mathrm{Xg}(\mathrm{a}+)$.

The serum creatine kinase levels were measured in 1973 and again in 1975. On both occasions a Boehringer Mannheim kit was used. The level in patient A (II.2) was 535 in 1973 and 205 in 1975. As the upper limit of normal is given as $50 \mathrm{mU} / \mathrm{ml}$, she is assumed to be a carrier. The level in patient $B$ was $12 \mathrm{mU} /$ $\mathrm{ml}$ in 1973 and $2 \mathrm{mU} / \mathrm{ml}$ in 1975 ; she is assumed to have a low probability of being a carrier.

The relative likelihood for value of $\theta$ indicates no detectable linkage between Duchenne muscular dystrophy and protanopia.

\section{Method used for calculating lod score}

The lod score $Z$ is defined $\log _{10} \frac{\mathrm{P}\left(\mathrm{F}_{1} \mid \theta\right)}{\mathrm{P}\left(\mathrm{F}_{1} \mid \frac{1}{2}\right)}$.

The first stage is to calculate the probability of obtaining the family $\mathrm{Pe}$ when the crossover rate is $\theta$.
Let $\mathbf{p}=\theta$ and $\mathbf{q}=1-\theta$ its complement.

There are three possibilities for the genotypes of generation I. Suppose these are designated A, B and C.

A-I.2 is a carrier for dystrophy and colour blindness in coupling,

B-I.2 is a carrier for dystrophy and colour blindness in repulsion.

$\mathrm{C}-\mathrm{I} .1$ is (a carrier for) colour blindness.

Let $\mathrm{D}$ represent the allele for normal and $d$ represent the allele for muscular dystrophy. Let $C$ represent the allele for normal vision and $\mathrm{c}$ represent the allele for protanopia.

Then the three possibilities of genotype in generation I can be tabulated as follows:

$$
\begin{aligned}
& \text { A }-I .2 \text { chromosomes DCdc, } \\
& \text { B-I.2 chromosomes DcdC, } \\
& \text { C-I.2 chromosomes DCdC, but I.1 Dc-Y. }
\end{aligned}
$$

Calculation of probability of obtaining pedigree of family Pe with possibility $A$

II.1 is normal and so has genotype DC-Y. No crossover has occurred, probability is q.

As he could have inherited the other maternal chromosome the total probability of him getting the genotype he did is $\mathrm{q} / 2$.

II. 2 is a carrier for dystrophy because of her creatine kinase levels. She may or may not also be a carrier for protanopia. Her genotype could be DCdc or DCdC, the initial probabilities of which are $q / 2$ and $p / 2$, according as to whether or not a crossover occurred.

Suppose she was DCdc, then if no further crossover occurred (q) her son who is normal (DC-Y) has a probability of $q / 2$. We will call this the subsequent probability.

On the other hand, if II.2 had the genotype DCdC the son has an equal chance of getting a normal chromosome whether or not a crossover ocurs. So the probability of III.1 is $\frac{1}{2}$. Subsequent probability is $\frac{1}{2}$.

So the total probability of Stem II.2 is the sum of 


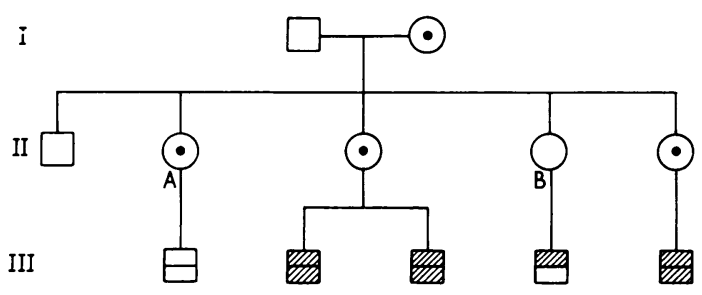

- Corrier by creatine kinose level or genetically

Protan colour blindness

Muscular dystrophy

Fig. Pedigree of family Pe.

The CK levels were done at the Area Laboratory at Taunton Hospital by courtesy of Dr J. Harkness.

the products of the initial and subsequent probabilities for the two possibilities for II.2's genotype.

Total probability of Stem II.2 is $\left(\frac{q^{2}}{4}+\frac{p}{4}\right)$.

II. 3 is calculated by the same method.

The only possible genotype for II.3 is DCdc.

Initial probability is $\mathrm{q} / 2$.

Subsequent probabilities are $\left(\frac{\mathrm{q}}{2}\right)^{2}$.

Total probability of II. 3 is $\mathrm{q}^{3} / 8$.

II. 4 is calculated again by the same method. She is not a carrier for dystrophy because of her normal creatine kinase levels. She is a carrier for protanopia as her son is affected. So her genotype is DCDc-a crossover has occurred. Initial probability of this stem is $p / 2$; subsequent probability of III. 4 is $\frac{1}{2}$. Total probability of this stem is thus $\mathrm{p} / 4$.

Finally II.5 could only have the genotype DCdc.

Initial probabilities are $\mathrm{q} / 2$.

Subsequent probabilities are $\mathrm{q} / 2$.

Total probability of stem II.5 is $\mathrm{q}^{2} / 4$.

The total probability of obtaining a pedigree is the product of all the sub-pedigrees and from $A$ it will be

$$
\left(\frac{q}{2}\right)\left(\frac{q^{2}+p}{4}\right)\left(\frac{q^{3}}{8}\right)\left(\frac{p}{4}\right)\left(\frac{q^{2}}{4}\right) .
$$

Calculation of probability of obtaining pedigree of family Pe with possibility $B$

Probability of II. 1 is $p / 2$ as a crossover has occurred.

Possible genotypes of II.2 are DCdc and DCdC.

Initial probability is $p / 2$ and $q / 2$.

Subsequent probability is $q / 2$ and $1 / 2$.
For stem II.2 total probability is $\left(\frac{\mathrm{pq}}{4}+\frac{\mathrm{q}}{4}\right)$.

Possible genotypes of II. 3 are only DCdc. A crossover must, therefore, have occurred. Probability is $\mathrm{p} / 2$.

Subsequent probability is $(\mathrm{q} / 2)^{2}$.

So the total probability for II. 3 is $\frac{\mathrm{pq}^{2}}{8}$.

Possible genotype for II.4 is DCDc. No crossover can have occurred.

Initial probability is $q / 2$.

Subsequent probability is $\frac{1}{2}$.

Total probability is $\mathrm{q} / 4$.

The only possible genotype for II.5 is DCdc, so a crossover must have occurred. Probability $\mathrm{p} / 2$.

Subsequent probability $\mathrm{q} / 2$. Total pq/4.

So the total probability of the pedigree Pe for possibility $B$ is

$$
\left(\frac{p}{2}\right)\left(\frac{p q+q}{4}\right)\left(\frac{p q^{2}}{8}\right)\left(\frac{q}{4}\right)\left(\frac{p q}{4}\right)
$$

Calculation of probability of obtaining pedigree of family $\mathrm{Pe}$ with possibility $\mathrm{C}$

In this case $\mathrm{I} .1$ is $\mathrm{Dc}-\mathrm{Y}$

$$
\text { I. } 2 \text { is DCdC. }
$$

Probability of II.1 is $\frac{1}{2}$ (crossing over is irrelevant here as I.2 is only heterozygous at one locus).

The genotype of II.2 is DcdC as she is a carrier of dystrophy because of her serum creatine kinase level. Probability is $\frac{1}{2}$ (again crossing over is irrelevant).

In order to have a normal son, III.1, a crossover must have occurred $\mathrm{p} / 2$.

Total probability $\mathrm{p} / 4$.

The genotype of II.3 must be DcdC. Her husband has normal colour vision, so we have to postulate subsequent crossovers to have her affected sons III.2 and III.3. Initial probability is $\frac{1}{2}$ (as for II.2)

Subsequent probability is $(\mathrm{p} / 2)^{2}$.

Total probability is $\mathrm{p}^{2} / 8$.

Genotype II.4 is DcDC. The Dc chromosome came from I.1 and the DC from I.2 and the chances of getting a normal chromosome from I.2 were equal whether or not a crossover occurred. By the same angument the subsequent probability of III.4 is $\frac{1}{2}$. Total probability is $\frac{1}{4}$.

Genotype of II.5 must be DcdC. Her husband is normal. So initial probability is $\frac{1}{2}$ and subsequent probability $\mathrm{p} / 2$.

So the total probability must be $\mathrm{p} / 4$. 
So the total probability of pedigree Pe for possibility $\mathrm{C}$ is

$$
\left(\frac{1}{2}\right)\left(\frac{p}{4}\right)\left(\frac{p^{2}}{8}\right)\left(\frac{1}{4}\right)\left(\frac{p}{4}\right)
$$

The total probability of getting the pedigree of the family $\mathrm{Pe}$ is $\frac{1}{3}(A+B+C)$.

The $\log$ likelihood for the family $L \theta=\log _{10}$.

$$
\frac{1}{3}(A+B+C)
$$

and the lod score $\mathrm{Z} \theta=\mathrm{L} \theta-\mathrm{L}\left(\frac{1}{2}\right)$

$$
=\log _{10}\left[\frac{\mathrm{pq}^{6}\left(\mathrm{q}^{2}+\mathrm{p}\right)+\mathrm{p}^{3} \mathrm{q}^{4}(\mathrm{pq}+\mathrm{q})+\mathrm{p}^{4}}{0.0742}\right] .
$$

For various values of $\theta$ these give lod scores as listed in the Table.

Table Lod scores for values of recombination fraction $\theta$ between 0.05 and 0.45

\begin{tabular}{lllll}
\hline$\theta$ & $\begin{array}{l}\text { For } \\
\text { family Pe }\end{array}$ & $\begin{array}{l}\text { Family L } \\
\text { Emery }(1966)\end{array}$ & $\begin{array}{l}\text { Total } \\
\text { lod score }\end{array}$ & $\begin{array}{l}\text { Relative } \\
\text { likelihood }\end{array}$ \\
\hline 0.05 & -0.325 & -0.442 & -0.767 & 0.171 \\
0.10 & -0.179 & -0.188 & -0.367 & 0.429 \\
0.15 & -0.158 & -0.062 & -0.220 & 0.603 \\
0.20 & -0.182 & 0.010 & -0.172 & 0.673 \\
0.25 & -0.220 & 0.051 & -0.169 & 0.678 \\
0.30 & -0.249 & 0.070 & -0.179 & 0.662 \\
0.35 & -0.246 & 0.073 & -0.173 & 0.671 \\
0.40 & -0.201 & 0.061 & -0.140 & 0.724 \\
0.45 & -0.114 & 0.037 & -0.077 & 0.838 \\
\hline
\end{tabular}

I am grateful to Dr Ruth Sanger, not only for doing the $\mathrm{Xg}(\mathrm{a}+)$ blood groups, but also for her help and advice. I also want to thank Professor Emery for drawing my attention to family $L$ and for letting me have his lod scores. The CK levels were done at the Area Laboratory at Taunton Hospital by courtesy of Dr. J. Harkness.

D. N. H. Greig,

The Surgery, Church'Square, Taunton, Somerset.

\section{Reference}

Emery, A. E. H. (1966). Genetic linkage between the loci for colour blindness and Duchenne type muscular dystrophy. Journal of Medical Genetics, 3, 92-95.

\section{$\mathrm{GM}_{1}$ gangliosidosis type 1 in twins}

SUMMARY This report describes 7-month-old monozygotic twin female infants with $\mathbf{G M}_{1}$ gangliosidosis type $I$. In addition to the usual clinical and biochemical abnormalities general- ized intracutaneous telangiectasis were present in both infants.

Generalized gangliosidosis, an inborn error of metabolism secondary to deficiency of the enzyme $\mathbf{B}$ galactosidase (Okada and O'Brien, 1968), is biochemically characterized by the visceral accumulation of a keratin sulphate-like mucopolysaccharide (Suzuki, 1968) in addition to the neuronal and visceral accumulation of a monosialonganglioside $\mathrm{GM}_{1}$ (ganglioside nomenclature of Svennerholm, 1964).

Clinically, two distinct phenotypes (type I and type II) of disease have been recognized, distinguished by age of onset and degree of visceral and skeletal involvement. Inheritance is autosomal recessive in pattern (Derry et al., 1968). In patients with type I disease neurological degeneration is progressive and despite good supportive therapy, survival past the age of 2 years is uncommon (O'Brien, 1969).

In type II disease the onset of neurological symptoms is later (7 to 16 months) and the coarsening of facial features, hepatosplenomegaly, and fundus changes characteristic of type I disease do not occur. Radiographic lesions, when present, are mild. Neurological degeneration is rapidly progressive with seizures occurring in approximately $50 \%$ of patients (Wolfe et al., 1970).

Definitive diagnosis is dependent on demonstration of a reduction in the enzyme $B$ galactosidase in peripheral leucocytes or in cultured skin fibroblasts. The enzyme assay is effective in the identification of both homozygous and heterozygous gene carriers and, in addition, has been successfully used for the prenatal diagnosis of afflicted infants (Nadler and Gerbie, 1970).

The occurrence of generalized gangliosidosis in twins has yet to be reported though the disease is well established as a distinct clinical and biochemical entity and has been the subject of several comprehensive reviews (Okada and O'Brien, 1968; Wolfe $e t$ al., 1970). This communication describes the occurrence of the infantile form (type I) of this disease in monozygotic twins.

\section{Case reports}

Seven-month-old twin Caucasian female infants were referred for examination of hepatosplenomegaly and psychomotor retardation. They were products of a 38-week uncomplicated pregnancy with normal labour and delivery. Gross and microscopical examination of the placenta revealed two amnions and one chorion.

Physical examination showed small, hypoactive 